\title{
Multiscale interpretation of contact problems
}

\author{
P. Brož \\ Professional Association for Science, \\ Research and Consultancy, Czech Republic
}

\begin{abstract}
Analysis of interactions between discrete and granular media demands larger computational mines. It is advantageous to employ for discrete systems with diffuse non-smoothness a domain-decomposition-like method. Concurrently, the numerical strategy is enriched by means of multiscale methodology to heal differences between discrete and continuum versions. To assess the said techniques, the balance of a tensegrity construction is selected. To express an idea of representative elements respective to the continuous medium homogenized variables are introduced.

The late developments in the numerical interpretation of large multicontact problems demand multiprocessor computers to obtain allowable computer time modellings. Contact stipulations result in non smooth mathematical formulations of steady-state and dynamical problems appearing from structural and granular mechanics. The examples of solvers as the Non Linear Gauss Seidel algorithm and the Conjugate Projected Gradient method were developed adaptable to a parallel treatment. The domain decomposition procedures permit one to go into large-scale mechanical problems, and their effectivity was demonstrated for linear tasks.

Keywords: beam, cable, granule, homogenization, non-smoothness, space frame, surface and volume representative elements.
\end{abstract}

\section{Introduction}

If variables in mechanical problems are independent between them, an easy distribution of the computations on different processors is effective; that implies that the problem is simply divided into many independent subproblems. The transition from large scale contact formulations to multilevel ones seems to be 
most fundamental for problems of multiscale discrete regimes; in particular papers [1] and [2] comprise this subject matter.

In structural mechanics, parallel computing strategies were applied to highly heterogenous construction such as fiber-reinforced composite or steel composite structures. For these scales, it is not simply to differ between structure and material.

While employed parallel computing in mechanics, if the contact exists on the boundary of a large scale domain before investigated a multiprocessor technique, no specific parallel strategy is formed. The contact may be handled in an outer iteration loop in a sequential manner, an inner iteration loop for analyzing the material problem demanding a parallel technique. When the contact occurs in many various areas, we refer to multicontact problems. It may further be of interest to define an especial multiprocessor strategy to link the contact stipulations with the other relations.

If the contact is the principal constitutive law of the system, we advise on diffuse non smoothness through all the structure or domain. The characteristic example is the granular medium viewed as a collection of rigid objects in interaction by contact and friction. However the tensegrity may be considered as diffuse non smooth regimes. Granular media are frequently large scale systems comprising several millions grains and have complex characteristics from a solid state to a dilute gas by way of a fluid flow. The modelling of such evolutionary problems demands parallel techniques to be carried out in a reasonable computing time. Several periods of the model software may profit from a parallel treatment, the balance or motion relations, but also the contact appearance.

Introducing multi-scale ways of tackling constitutes an incentive to improve the solution to contact problems. Two principal strategies in multiscale techniques for solid mechanics problems are bottom-up which represent start-up from first principles and further coarsening the outcomes at the next level and top-down when macroscopic models are got from simulations depicting the physical state at lower scales. Bottom-up methods are based on statistical mechanics. Top-down approaches apply homogenization and relaxation. Sequential or information-passing procedures are used also as concurrent or laid down scale overcoming for the formulation of multi-scale schemes. According to [3], in the sequential or information-passing systems, the discrete scale is simulated and its response is introduced into the next scale by means of homogenization while in the cooperating method the various scales are simultaneously resolved. For information-passing multi-scale methods the scales both of time and spatial must be partible. Concurrent scale bridging procedures resolve multiple scales simultaneously at different parts of a construction. In this regard it is desirable to labour a formulation of representative elements in greater detail, in conformity with Continuum Damage Mechanics, e.g. [5].

Micro-mechanical contact at the interface surface is taken into consideration. This is linked to the extraordinary complicated construction at the surface texture and the chemical and physical characteristics of the material nearly the interface. Hitherto these qualities are contemplated by using macroscopic constitutive relations at contact interfaces similar to the classical Coulomb law. But still 
detailed analysis that provides better physical glimpse or optimization of engineering structures demands multi-scale concept. When proceeding to lower scales it is necessary to consider three-dimensionality in order that describing the processes on these scales appropriately and to be capable to read quantitative issues.

Constitutive equations for the micro-mechanical outlook on two contacting bodies within the contact area provides response function for the normal and tangential stresses as regards parameters such like average real area of contact, the prevalent mean plane distance or relative velocities. Constitutive equations for the normal contact area set up by seeing into micro-mechanical characteristics in the contact surface.

Generally, the micro-mechanical behaviour depends on material parameters as hardness and upon geometrical parameters as surface roughness. But the micro-mechanical effects are extraordinary complex owing to very high local pressures at the unevennesses and the potential presence of finite deformation at this level. Consequently the simulations employed for this type of analysis have to contain nonlinear material characteristics and large strains. In [4] a finite strain model was used to calculate from numerical modellings a constitutive relation for the interpretation at two deformable surfaces. For the interfacial characteristics related to the frictional response, the most often used constitutive equation is the classical law of Coulomb. But, other parameters affect the frictional behaviour alike relative velocity temperature, constitutive characteristics at the contacting objects and roughness. As a result, knowledge of these parameters is necessary to model the contact behaviour at micro-scale.

\section{Idea of multi - scale modelling}

Normal contact can be often simulated by applying the classical constraint relations of non-penetration (opening $g_{N} \geq 0$ ) and the presence of only compressive stresses at the contact interface (normal stress $t_{N} \leq 0$ ). These stipulations can be reformulated in Kuhn - Tucker form.

Coulomb law is frequently sufficient to model the characteristics for tangential contact. Concurrently, the relative tangential velocity is divided into a stick and a sliding part. Also the stick portion represents a constraint like in normal contact, the sliding part is determined by a constitutive equation that in its simplest form implies the law of Coulomb to a constant friction coefficient $\mu$. If the material characteristics are more complicated at the contact interface, subsequently it may be urgent to take the microstructure of the contacting surfaces into consideration. Instinctively, this microstructure is of a diverse length scale than the scale of the engineering problem in which contact exists. E.g. for the tyre footprint, the length scale [cm] to [m] is recommended whereas the necessary scales to be used to simulate the road surface are in the micrometer zone $[\mu \mathrm{m}]$. In the case of some effects as adhesion it may be even urgent to switch to smaller scales nanometer. Here the influences related to the micro-scale within a contact problem that must be analyzed on a macro-scale level. To solve this, several concepts were found. The accent is put on the sequential multi-scale 
approach that is applied together with statistical evaluations. Consequently scale separation of the physical mechanisms is supposed to be true to life for the normal and tangential characteristics of the contact interface. Within the bounds of this methodology homogenization of micro-scale issues with regard to the next scale has to be performed and the corresponding homogenization routines must be elaborated on. They are construed in such a manner that the outcomes of a micro finite element model introduce averaged values for the constitutive characteristics on the next scale.

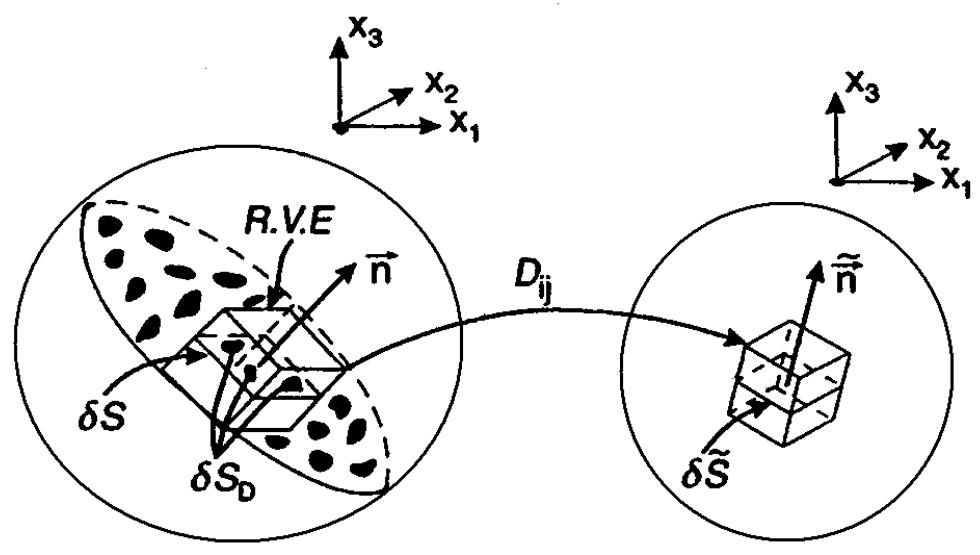

Figure 1: $\quad$ Damage in physical and mathematical sense.

In engineering, the mechanics of continuous media introduces a Representative Volume Element (RVE) on which all properties are represented by homogenized variables. Concurrently, a repetitious construction is the matter. To give an order of magnitude, its size can vary from about $(0.1 \mathrm{~mm})^{3}$ for metals and ceramics to about $(100 \mathrm{~mm})^{3}$ for concrete. The damage discontinuities are "small" with regard to the size of the RVE but of course large compared to the atomic spacing. From physical point of view, damage is always related to plastic or irreversible strains and more generally to a strain dissipation either on the mesoscale, the scale of the RVE, or on the microscale, the scale of the discontinuities. For a larger generality where microcavities and microcracks may exist, the damage variable is physically defined by the surface density of microcracks and intersections of microvoids lying on a plane cutting the RVE of cross-section $\delta S$. For the plane with normal $\vec{n}$ where this density is maximal, we have

$$
D_{(\vec{n})}=\frac{\delta S_{D}}{\delta S}
$$

For quasi-brittle failures or high cycle fatigue, for which the loading is below the convectional yield stress, plasticity (or dissipation) and damage occur but at a scale much smaller than the RVE scale. A multi scale approach is thus necessary. 
Two scales are defined in accordance with growth of microscale damage in a mesoscopic RVE:

- The mesoscale or the classical scale of continuum mechanics

- The microscale or the scale of microdefects present inside the RVE but whose effects on the mechanical properties are not measurable, except for monotonic and fatigue rupture properties. From the mechanical point of view of the homogenization procedures, such defects are considered altogether as a "weak" inclusion in a RVE matrix.

The RVE definition is applied even if such a representative component is not all the time easy to explain. So far for granular materials this definition is an open question since the medium is understandably discreet and the interaction between constituents are complicated. For discrete structures be may without difficulty differentiate a basic element or module.

STRUCTURE CALCULATION

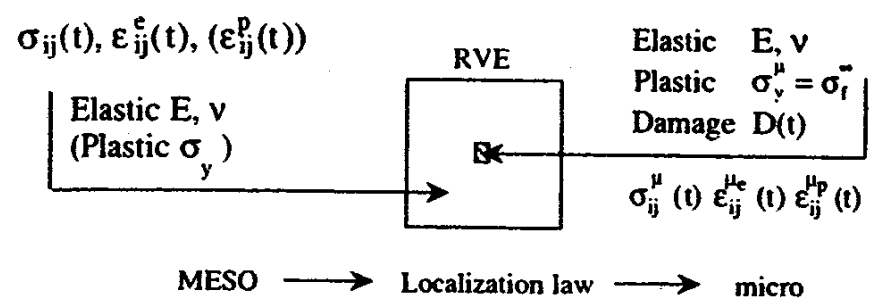

Figure 2: Microelement laid down in an elastic (plastic) RVE.

In comparison with the RVE, it is suitable to compute averaged values for the normal and tangential stresses by projecting the local stress fields onto the directions formulated on the boundary of a Representative Surface Element (RSE). The boundary of the RSE is defined by the normal vector $\mathbf{n}^{0}$ and the vectors $\mathbf{a}_{\alpha}{ }^{0}$. The related surface zone is expressed by $\Gamma_{c}{ }^{0}$. The local geometry at micro level is stipulated by the normal vector $\mathbf{n}^{i}$ and the tangent vectors $\mathbf{a}_{\alpha}{ }^{i}$. They vary from one local contact area $i$ to the next. Concurrently, the contact surface in denoted by $\Gamma_{c}^{i}$ and the normal and tangential stresses are calculated through a micro finite element analysis ending in the local normal stress $t_{N}{ }^{i}$ and the local tangential stress vector $\mathbf{t}_{T}{ }_{T}$. These stresses can be employed to calculate in the average stresses by the integration of all local contact surfaces at micro-scale inside the RSE. For averaged (effective) normal stress $\left\langle t_{N}\right\rangle$ it holds

$$
\left\langle t_{N}\right\rangle=\frac{1}{\Gamma_{c}^{0}} \sum_{i} \int_{\Gamma_{c}^{i}}\left(t_{N}^{i} \mathbf{n}^{i} \cdot \mathbf{n}^{0}+\mathbf{t}_{T}^{i} \cdot \mathbf{n}^{0}\right) d \Gamma
$$

Take notice of local tangential stresses are also instrumental in the normal stress. Predictably for a enough large RSE, these stresses will often vanish. Nevertheless for specific microstructures they can play a part. Like for the 
normal stresses also the averaged value of the constituents of the tangential stresses $\left\langle\mathbf{t}_{T}{ }^{0}\right\rangle=\left\langle t_{T}{ }^{\alpha}\right\rangle \mathbf{a}_{\alpha}{ }^{0}$, according to [3] may be calculated from

$$
\left\langle t_{T}^{\alpha}\right\rangle=\frac{1}{\Gamma_{c}^{0}} \sum_{i} \int_{\Gamma_{c}^{i}}\left(t_{N}^{i} \mathbf{n}^{i} \cdot \mathbf{a}^{0 \alpha}+\mathbf{t}_{T}^{i} \cdot \mathbf{a}^{0 \alpha}\right) d \Gamma
$$

for $\mathbf{a}_{\alpha}{ }^{0} \cdot \mathbf{a}^{0 \beta}=\delta_{\alpha}{ }^{\beta}$.

\section{Mathematical statements of multicontact problems}

Very wide-ranging contact cases are in accordance with the variety of the numerical algorithms to solve the relations modelling the contact conditions. In compliance with the link in which the numerical tools are evolved, the contact interactions may be highly dissimilar. At the outset, the contact comprises inequalities. Brief formulations practical for the mathematical analysis apply to the Convex Analysis, above all to the subdifferential calculus of convex functions. We fix our attention on a representative problem involving unilateral constraints resulting in distinct mathematical formulations, leading to acquire different effective numerical strategies. The said problem being pertinent to a structural sort issue, contains a deformable construction.

\subsection{Static structural problem}

The so called structural type problem lies in specifying the equilibrium of an elastic body in grazing contact with a stiff foundation. As a first sign of this case, the contact performs as a boundary condition. We frankly contemplate the discrete case using finite element method: $n_{n}$ means the total number of nodes, $n_{c}$ the number of potential contact nodes. In the first approximation, the equilibrium equations comprise a linear system: stiffness matrix $\mathbf{K}$, generalized displacement $\mathbf{u}$, contact reactions $\mathbf{r}$ acting on the nodes of the contact zone, the external forces f,

$$
\mathbf{K u}-\mathbf{H r}=\mathbf{f}
$$

The matrix $\mathbf{H}$ changes over from the local frameworks to the global one for all potential contact nodes. We must append, all the contact conditions expressed here with two inequalities and a supplementary condition between the contact reactions and the normal constituents of the displacements on the potential contact surface.

$$
\mathbf{H}^{t} \mathbf{u} \geq 0, \mathbf{r} \geq 0, \mathbf{r} . \mathbf{H}^{t} \mathbf{u}=0 \Leftrightarrow 0 \leq \mathbf{H}^{t} \mathbf{u} \perp \mathbf{r} \geq 0
$$

Frictional stipulations may make whole this system without alternating the main characterizations of the problem except the non symmetry which may be caused by a Coulomb type law. Consequently, the number of contacts $n_{c}$ is small in comparison with the total number of nodes denoted by $n_{n}: \mathbf{K}$ is a $2 n_{n} \times 2 n_{n}$ or $3 n_{n} \times 3 n_{n}$ matrix according to the two-dimensional or three-dimensional 
modelling; $\mathbf{H}$ is a $2 n_{n} \times n_{c}$ or $3 n_{n} \times n_{c}$ matrix. At length, the stiffness matrix is not easily invertible and it is useful to keep the global variable $\mathbf{u}$ as a main unknown of the problem. All the more thus as other non linearities in addition to contact can exist in such structural problem: the large deformations, e.g. cellular material, or nonlinear characteristics like the plasticity in metal forming. To tackle the system (4)-(5) with pertinently other non linearities, a Newton similar method seems as a general non linear solver even if it must be extended to non differentiable equations.

\subsection{From a truss to a granulate through the tensegrity}

Static and dynamic simulations of a structure being constituted of bars and cables are the following. Such a discrete construction may be described as a set of nodes and connection between them, the non smoothness only existing in the constitutive relations of the links. In the set of nodes $\Omega$, we make different the subset $\Gamma_{u}$ of the nodes where the displacement is decreed to grip the construction to the support. Three sets are to be contemplated: the prevalent one $\Omega_{1}$ for which the tensions and displacements are unknown, the prestressed configuration $\Omega_{0}$ before applying additional external loading and the relaxed configuration $\Omega_{-1}$ for which the selfstresses are virtually disappeared. The three configurations are supposed to be closed enough to protect the principle of small perturbations and the prestresses are assumed to be known.

\subsubsection{Basic equations}

For smooth movements the dynamical equation involves the time-derivative of the velocities. As impacts are expected, it is more practical to name this equation as the so called measure differential equation

$$
M d v+B^{t} \tau d v=F^{d} d t
$$

where $d t$ is a Lebesgue measure, $d v$ is a differential measure symbolizing the acceleration, $d v$ a non-negative real measure relative to which $d v$ occurs to possess a density function, and $\tau$ is a stand-in of local density of tensile forces. The balance equation lies in neglecting the inertial term and the measures.

$$
-F+F^{d}=0 \text { with } F:=B^{t} \tau
$$

A dual (or reduced to links) formulation of the dynamic may be favoured using the Delassus operator $W$

$$
d \eta-W \tau d v=-\bar{F}^{d} d t=-B M^{-1} F^{d} d t
$$

When a time discretization is carried out, an elementary subinterval $\left[t, t^{+}\right]$of length $h$ is taken into account. Since discontinuous velocities are awaited, high order integration schemes are not essential and even difficult; first-order schemes are adequate when many shocks appear simultaneously. We contemplate a 
completely implicit scheme underlining the impulsion $\pi$ over the time step as the product of $h$ by an average tension $\tau^{+}$regarded at the end of step,

$$
\eta^{+}-\eta^{-}-W \pi=-h \bar{F}^{d} \text { with } \pi=h \tau^{+}
$$

For statics, the strain admissibility equation joints the nodal displacements to length variations of the connections; for dynamics the strain rate admissibility equation connects the nodal velocities to relative velocities of the ends of the links.

There are intermediate items of the algorithm of application in question: (i) constitutive laws, (ii) space frames, and (iii) net and granules.

\subsubsection{Tensegrity}

In general, the stiffness of the cables is weaker than in the case of bars. It is then useful to contemplate extensible cables instead of inextensible ones. Such a construction is a selfstressed tensegrity system if the set of compressed components is discontinuous and the assortment of tensioned components is continuous. The matrix $K_{b}$ is in that case singular with a large kernel put together of the rigid modes of all the bars. As the global stiffness of the joined truss is invertible, the equilibrium of the system may be characterized by a linear complementary problem entailing the tensions in the cables and the corrected length modifications

$$
\left\{\begin{array}{l}
A_{c} \lambda_{c}-\tau_{c}=-k_{c} B_{c} K^{-1} F^{d}-\tau_{c}^{0} \\
0 \leq \lambda_{c} \perp \tau_{c} \geq 0
\end{array} \quad \text { with } A_{c}=k_{c}-k_{c} B_{c} K^{-1} B_{c}^{t} k_{c}\right.
$$

The one step dynamical discrete problem is a Linear Complementary Problem as formerly, considering the corrected velocities $\lambda_{c}{ }^{+}$.

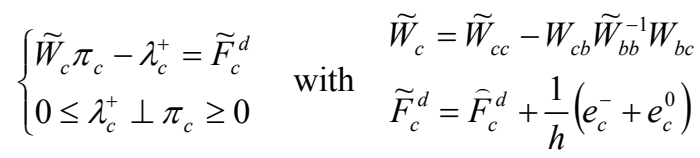

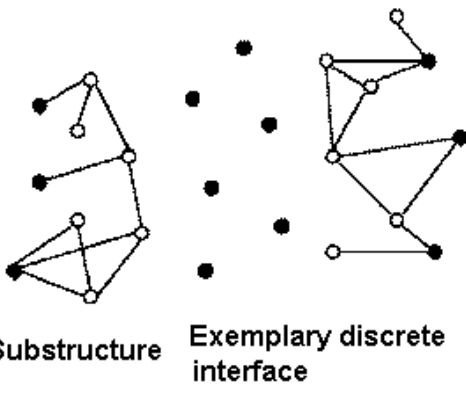

Figure 3: Discrete interface. 


\section{Substructuring strategy}

First, reaching the reformulation of the problem lies in a decomposition of the construction into interfaces and substructures (fig. 3). Each of these constituents owns its variables and equations. In so doing, connections are spread among substructures, and interfaces are nodes linking one substructure to another. A substructure $E$ is subject to the acting of its adjacent interfaces $\Gamma_{E E^{\prime}}$ : forces $F_{E E^{\prime}}$ and displacements $U_{E E^{\prime}}$. Augmented to whole interfaces local to the substructure $E$, the collection of the former fields are indicated with $F_{E \Gamma}$ and $U_{E \Gamma}$. An interface $\Gamma_{E E^{\prime}}$ passes the forces $F_{E E^{\prime}}$ and the displacements $U_{E E^{\prime}}$ on each of its faces.

The solution $s=\cup_{E} s_{E}$ when $s_{E}=\left(e_{E}, t_{E}, U_{E E^{\prime}}, F_{E E^{\prime}}\right)$ of the reference problem must fulfils:

- the equilibrium equation

$$
-F_{E}+F_{E}^{d}+C_{E \Gamma}^{l} E_{E \Gamma}=0
$$

where $F_{E}=B_{E}^{l} t_{E}$;

- the strain acceptability

$$
\left\{\begin{array}{l}
U_{E \Gamma}=C_{E \Gamma} U_{E} \\
e_{E}=B_{E} U_{E} \\
\left.U_{E}\right|_{\Gamma_{u}}=U_{E}^{d}
\end{array}\right.
$$

- the constitutive relations (14) and (15)

$$
\begin{gathered}
t_{b}+t_{b}^{0}=k_{b}\left(e_{b}+e_{b}^{0}\right) \\
\left\{\begin{array}{l}
\tau_{c} \geq 0 \\
\lambda_{c} \geq 0 \\
\left(\tau_{c}\right)^{t} \lambda_{c}=0
\end{array}\right.
\end{gathered}
$$

- the interface characteristics

force equilibrium:

$$
F_{E E^{\prime}}+F_{E^{\prime} E}=0
$$

continuity of displacements:

$$
U_{E E^{\prime}}=U_{E^{\prime} E}
$$


The interfaces show exemplary characteristics due to both the continuity of displacements and the non-smoothness that is located within the substructures. As a test occurrence is contemplated a tensegrity gridwork got by the duplication a self-stressed simple module. Such a module is constituted of 8 nodes, 12 cables and 4 bars. This prestressing brings about that the module becomes self-stressed (the product of the note to link mapping by prestress), ie. $B t^{0}$. That is why any configuration of such modulus will be unintentionally self-balanced. The experimented tensegrity grid has $16 \times 16=256$ modules, it is divided into $4 \times 4$ $=16$ substructures (each holding 16 modules) and 24 sturdy interfaces. To compare, an alike substructuring of a continuum media plate would result in 16 substructures and 24 interfaces solely, not one of them as "weak". Being boundary conditions, the lower nodes on two opposite edges are fixed and a unified vertical force domain $F^{d}=40 \mathrm{~N}$ is decreed at every node.

In the case of the test event thought about, the loading level is near to, yet less than, its ultimate limit value, and there is a deficiency of stability of the entire structure (like to global buckling in continuum media and applicable to the mechanism existing here). Several loading amplitudes $\alpha F_{d}$ for $0 \leq \alpha \leq 1$ are taken into account. For these values of $\alpha$, the modelling is carried out in one step from the reference configuration $\Omega_{0}$ to the present one $\Omega_{\alpha}$, without time stepping. This can be achieved for the considered problem as it is not an evolution-type simulation: the last solution does not depend on the loading path.

The values of $\tau_{c}$ are indicated in fig. 4 on each of the 3072 cables, for the various values of $\alpha$, classified by an increasing order.

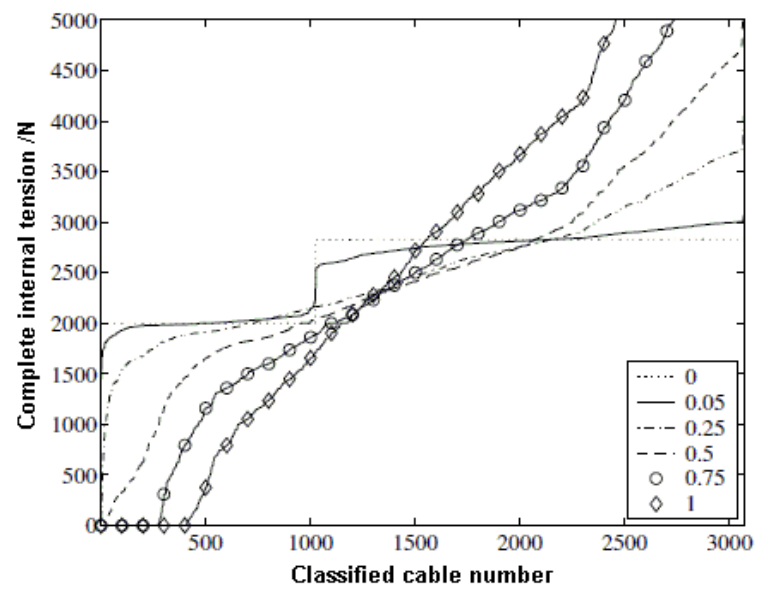

Figure 4: Internal tension in cables for various values of the loading parameter $\alpha$.

For $\alpha=0$, the values $\tau_{c}^{0}$ in the cables are got back. Evidently, when $\alpha$ rises, the stress redistribution is growing increasingly (the maximum value of internal tensions $\tau_{c}$, too) to achieve around $14 \%$ of all the set of cables if $\alpha=1$. In the case of this loading value, the construction is still acting within its stable field 
and it still owns further a stiffness reserve. Similar simulation are practical to examine the integrity of such a structure subject to extreme loading condition above normal service practice when, generally, one assesses that no cable slacks off. At the same time, the strength of the construction could be put at risk if the load decreases another time and when slackened cables unexpectedly reload: the fast alteration in local obvious stiffness ends in dynamical loading that can harm the nodes.

\section{Notes to foregoing methodologies}

\subsection{Domain decomposition approach}

Domain decomposition concept was worked in computational structural mechanics out to solve large-scale problems discretized by the finite element method. The substructuring procedures are effective techniques for their permit to reduce memory storage and computing time. Moreover these methods take advantage of the new multi-processor generation of computers as they exhibit an intrinsic parallelism with a high granularity. The main component of the domain decomposition algorithm is a numerical solver based on the solution of local independent subproblems on subdomains. In addition, these methods are efficient solvers in a classical monoprocessor environment, too. In [1], two domain decomposition approaches introduced for linear systems are investigated and two strategies to solve large-scale multicontact problems are discussed.

\subsection{Multiscale approach for diffuse non smoothness}

Substructuring seems to be an understandably multiscale numerical method when each substructure is linked to an intermediate scale between the basic constituents within the subdomains and the global construction. Besides multiscale approaches can enhance the substructuring in affiliating either a micro-macro division of some variables or even a homogenization routine. Via this way the domain decomposition approaches represent not only efficient numerical methods to solve large-scale problems but may also provide a numerically homogenized behavior of each substructure which is useful from a mechanical point of view. An attempt is presented to extend the LATIN (Large Time Increment) micro-macro approach, initially developed for continuous media to strongly non-smooth discrete systems. This method is used to the tensegrity construction whose equilibrium is marked by the Linear Complementary Problem.

\section{Conclusion}

The method presented works at large scale non-smooth problem tackled by a substructuring technique. To solve discrete systems with diffuse nonsmoothness, a multiscale algorithm is employed. The applicability is tested for quasi-static characteristics of tensegrity construction subject to very loading, 
with a substantial quantity of slackened cables. Multilevel domain decomposition algorithms are anticipated to demonstrate numerical gradability regarding the number of subdomains. This is not relevant to the height of diffuse non-smoothness. Modifications are worked out to get over this inconvenience.

There are some views in this subject desirable to find a solution, among other things, concerning dynamical characteristics of tensegrity structures, granular media models, and a prospect to heal the difference between the discrete simulation and the continuum macro model of structures.

The contact interpretation in a multiprocessor depends on the problem in which the contact exists, in the main on the ratio of degrees of freedom involved by non-smooth contact stipulations. When the contactless problem joined with contact one demands a parallel computing strategy, the multiprocessor concept to treat with contact must fit the former strategy. In so doing, the contact area may be localized in the subdomain or on the interfaces between the subdomains. The parallel computing may lie in extending linear solvers to non linear and even non smooth ones. When considering the contact treatment, a more general parallel computing strategy is applied to solve non linear large scale problems.

\section{Acknowledgement}

The author gratefully acknowledges the financial support of the presented research by the Grant Agency of the Czech Republic (project No. 103/09/1580).

\section{References}

[1] Alart, P., Contact on Multiprocessor Environment: from Multicontact Problems to Multiscale Approach. In: Computational contact mechanics, Courses and lectures - No. 498, Eds P. Wriggers and T. A. Laursen, pp. 163-218, Springer Wien, New York, 2009.

[2] Nineb, S., Alart, P., Dureisseix, D., Domain decomposition approach for non-smooth discrete problems, example of a tensegrity structure. Computers and Structures, 85, pp. 499-511, 2007.

[3] Wriggers, P., Nettingsmeier, J., Homogenization and Multi - Scale Approaches for Contact Problems. In: Computational contact mechanics, Courses and lectures - No. 498, Eds P. Wriggers and T. A. Laursen, pp. 129-162, Springer Wien, New York, 2009.

[4] Bandeira, A. A., Wriggers, P., de Mattos Pimenta, P., Homogenization methods leading to interface laws of contact mechanics. International Journal for Numerical Methods in Engineering, 59, pp. 173-195, 2004.

[5] Lemaitre, J., A Course on Damage mechanics, Springer, Berlin, Heidelberg, New York, 1992-1996.

[6] Brož, P., Procházka, P., Contact Problem of Two Elastic Bodies. Acta Technica ČSAV, No. 6, pp. 608-613, 1985.

[7] Danek, J., Hlavacek, I., Nedoma, J., Domain decomposition for generalized unilateral semi-coercive contact problem with given friction in elasticity. Math. And Comp. in Simulation, 68, pp. 271-300, 2005. 